\title{
Evaluation of the Areas Involved in Visual Cortex in Parkinson's Disease Using Diffusion Tensor Imaging
}

\author{
Somayeh Mohammadi Jooyandeh ${ }^{1}$, Aida Kamalian ${ }^{2}$, Sepideh Shiranvand ${ }^{2}$, \\ Mahsa Dolatshahi ${ }^{2}$, Mohammad Hadi Shadmehr ${ }^{2}$, Thomas C. Baghai ${ }^{1}$, \\ Farzaneh Rahmani ${ }^{2}$, Ahmad Shojaie ${ }^{3}$, and Mohammad Hadi Aarabi ${ }^{3, *}$ \\ 1 Department of Psychiatry and Psychotherapy, University of Regensburg, \\ Regensburg, Germany \\ 2 Students'Scientific Research Center,Tehran University of Medical Sciences, Tehran, \\ Iran \\ 3 Basir Eye Health Research Center, Tehran, Iran \\ Mohammadhadiarabi@gmail.com
}

\begin{abstract}
Parkinson's disease (PD) is a progressive neurodegenerative disorder assumed to involve different areas of CNS and PNS. In PD patients and in primates with experimental Parkinsonism indicating that retinal dopamine deficiency is an important factor in the pathogenesis of PD visual dysfunction. Visual signs and symptoms of PD may include defects in eye movement, pupillary function, and in more complex visual tasks. In this study, we evaluated the areas involved in visual cortex in $\mathrm{PD}$ by diffusion tensor imaging to assess the structural change in PD.
\end{abstract}

Keywords: Diffusion tensor magnetic resonance imaging, Connectomics, Parkinson disease and Visual Cortex.

\section{Introduction}

Parkinson's disease (PD) is a common neurodegenerative disorder affecting middle aged and elderlypeople.It is a disease defined with deficiency of dopamine in areas of the midbrain causing a variety of movement problems such as shaking, rigidity, slowness of movement and difficulty with walking and gait. Sensory problems of PD may include visual loss, loss of smell, auditory problems [1]. In PD patients and in primates with experimental Parkinsonism retinal dopamine deficiency is an important factor in the pathogenesis of PD visual dysfunction. Visual signs and symptoms of PD may include defects in eye movement, pupillary function, and in more complex visual tasks involving the ability to determine interval of the shape of an object and visual hallucination $(\mathrm{VH})[2,3]$. The findings show that basic visual sensory function, and visual perception are impaired also in nonmanic patients with mild to moderate PD. Among numerous advanced MR techniques Diffusion-weighted image (DWI) and diffusion tensor imaging (DTI) are methods that have been used in recent years to find changes of white

X. Chen, M. K. Garvin, J. Liu, E. Trucco, Y. Xu (Eds.): OMIA 2016, Held in Conjunction with MICCAI 2016, Athens, Greece, Iowa Research Online, pp. 145-151, 2016. Available from: http://ir.uiowa.edu/omia/2016_Proceedings/2016/ 
matter integrity because of their sensitivity to the local diffusion behavior of water molecules in brain tissue [4]. The utilization of DWI and DTI have proved to be effective in differential diagnosis of PD and Parkinson variants, such as Multiple system atrophy and progressive supranuclear palsy [5]. So far, DTI has been widely practical for the quantitative evaluation in many types of brain disease, including amyotrophic lateral sclerosis [6], schizophrenia [7], Parkinson's disease [8], NMO [9], and multiple sclerosis [10], and it has also been used in the quantitative evaluation of the optic nerves in normal brain [11], idiopathic demyelinating optic neuritis [12], and indirect traumatic optic neuropathy [13]. Other imaging techniques including PET and functional MRI studies have shown reduced activation of the ventral/lateral visual association cortices and the primary visual cortex (VC) in particular [2]. While few studies have been done on DTI.

In our study, we aimed to calculate healthy and PD visual cortex brain connectivity matrices for average tract length and average fiber volume and compare them directly through Network Based Statistics (NBS).

\section{Method}

\subsection{Participants}

Participants involved in this research were recruited from Parkinson's Progression Markers Initiative (PPMI) [14]. DWI images were obtained for 18 patients (7F $11 \mathrm{M}$, mean age 73.33) and 12 controls (4F 8M, mean age 71.50). Participants were tested and confirmed negative for any neurological disorders apart from PD. The participants' PD status was confirmed by Movement Disorder Society-Unified Parkinson's Disease Rating Scale (MDS-UPDRS) and the loss of dopaminergic neurons were observed in DaTscans. Every participant involved in this research has signed informed written consents in order to share their unidentified clinical data to investigators.

\subsection{Data Acquisition}

Data used in the preparation of this paper was obtained from Parkinson's Progression Markers Initiative (PPMI) database (www.ppmi-info.org/data/) [14]. This dataset was acquired on a 3 Tesla Siemens scanner, producing 64 DWI (repetition time $=7748 \mathrm{~ms}$, echo time $=86 \mathrm{~ms} ;$ voxel size: $2.0 \times 2.0 \times 2.0 \mathrm{~mm}^{3}$; field of view $=224 \times 224 \mathrm{~mm}$ ) at $\mathrm{b}=1000 \mathrm{~s} / \mathrm{mm}^{2}$ and one b0 image along with a 3D T1-weighted structural scan (repetition time $=8.2 \mathrm{~ms}$, echo time $=3.7 \mathrm{~ms}$; flip angle $=8^{\circ}$, voxel size: $1.0 \times 1.0 \times 1.0 \mathrm{~mm}^{3}$; field of view $=240 \mathrm{~mm}$, acquisition matrix $=240 \times 240)$.

\subsection{Diffusion MRI Data Processing and Network Construction}

The DWI data were analyzed and processed in ExploreDTI [15]. The diffusion tensor was estimated using a weighted linear least square regression procedure 
proposed in [16]. For subject motion correction and eddy-current induced geometrical distortion, DWI data were rigidly registered with MNI atlas [17]. During this processing step, we adjusted the B-matrices with appropriate reorientations and included the required signal intensity modulation with the Jacobian determinant of the spatial transformation [18]. Whole-brain tractography was performed using a deterministic algorithm.

Fibers were reconstructed by placing seed points on a uniform grid across the data set at $2 \mathrm{~mm}$ isotropic resolution and by following the main diffusion direction (as defined by the principal eigenvector) unless the fiber tract entered a voxel with a $\mathrm{FA}<0.2$ or it made a high angular turn considered to be anatomically implausible (angle $>30^{\circ}$ ). The step size was set at $0.5 \mathrm{~mm}$. The whole-brain fiber tracts were then parcellated using the automated anatomical labeling (AAL), a widely used atlas to derive nodes in graph theoretical analyses of neuroimaging data [19]. The mentioned atlas divides brain into 6 cortical regions. The interregional connectivity between the 6 ROIs demarcated on the AAL template was computed by applying the ROI masks to the reconstructed fiber tracts using ExploreDTI. In this fashion we determined the volume and average tract length that originated in one ROI (i) and terminated in another one (j), for all 6 ROIs defined on the atlas, creating a $6 \times 6$ connectivity matrix $(\mathrm{CM})$.

In the next step we used NBS for group analysis [20]. NBS is a method based on the principles underlying traditional cluster-based thresholding of statistical parametric maps to control family-wise error rate (in the weak sense) when mass-univariate testing is performed at every connection of the graph. The gain in power offered by NBS increases as the number of nodes and links is increased, which serves well in our complex neural network. In brief, by using NBS, the presence of every link is utilized to yield greater power than a generic procedure to control FWER.

\section{Result}

T-test statistics were applied with threshold of 2.9 and significant results (pvalues for average tract length was 0.0202 (Table 1) (Figure 1).The NBS identified two subnetworks of reduced connectivity in de novo $\mathrm{PD}$ patients $(\mathrm{P}<0.05$, corrected for multiple comparison).

Table 1. The Significant Pathway

\begin{tabular}{|c|c|}
\hline Pathway & Test Stat \\
\hline Calcarine Right to Lingual Left & 2.01 \\
\hline Calcarine Right to Calcarine Left & 2.32 \\
\hline
\end{tabular}



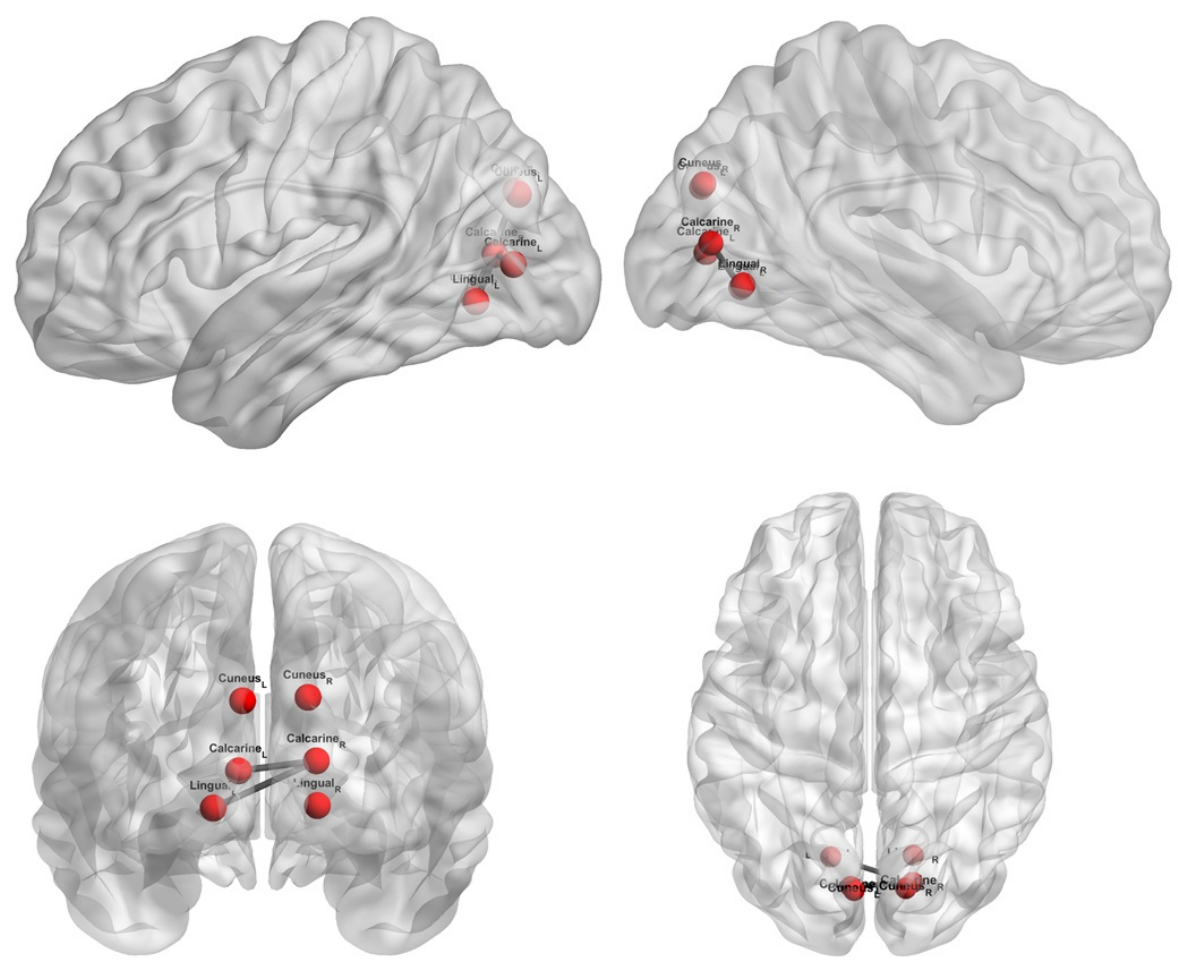

Fig. 1. Graphical illustration of significantly altered connectome pathway: average tract length

\section{Discussion and Conclusion}

In this study, for the first time, we explore occipital lobe of Parkinson's patients using DTI techniques, Our results suggest that several areas of visual cortex invoved in PD including:calcarine (left,right), lingual(left,right), cuneus that percent of distruption of left calcarine to right calcarin/ right calcarine to lingual was more than other areas, and also we show that visual dysfunction can occur in parkinson's patient and Affect their lives ,so detection of visual symptoms is important for differential diagnosis and patient management.

Regions of increased activation during the visual hallucinations relative to non-hallucination events included the bilateral cingulate gyrus (anterior, middle, and posterior), bilateral insula (including the claustrum), right medial frontal gyrus, right postcentral gyrus, right thalamus (near the medial dorsal nucleus), and right brainstem (near the red nucleus).Regions of decreased activation during the visual hallucinations relative to non-hallucination periods included the visual system on the right side (inferior occipital gyrus, lingual gyrus, and fusiform gyrus), the right cingulate gyrus, right superior temporal gyrus and the left 
middle frontal gyrus. There was no overlap between regions of increased and decreased activation during the visual hallucination events [21].

In $\mathrm{PD}$, the DMN showed reduced connectivity during the resting state, compared to controls. However, connectivity within the PDVH group was greater than the PD nonVH group, particularly across bilateral precuneus/posterior cingulate gyrus and right middle frontal lobe. The decreased functional connectivity within DMN in PD compared to controls is in line with a recent study showing that cognitively unimpaired patients have decreased functional connectivity within the DMN compared to controls [22]. Individuals with greatest hallucination symptoms actually had "higher" connectivity within the disrupted network, which in turn may increase functional connectivity across distant brain regions.

PD patients exhibited decreased short-range functional connectivity densities in regions that were mainly located in the ventral visual pathway and decreased long-range functional connectivity densities in the right middle and superior frontal gyrus, which have been speculated to be associated with visual hallucinations and cognitive dysfunction, respectively. The observed functional connectivity density alterations might be related to the disturbed structural connectivity of PD patients, leading to abnormal functional integration [21]. Morphometric studies based on structural MRI have also documented significant reductions in graymatter volume, cortical thickness, and cortical gyrification, as well as subcortical volumetric atrophy, in PD patients [23].

In a study, we observed focal reductions in both the short- and long-range FCDs of PD patients compared with the healthy controls. Notably, the regions with short-range FCD reduction were mainly located in the ventral visual pathway involvingthe inferior and middle occipital gyrus, Brodmann's area 18 and 19, lingual and fusiform gyrus, and inferior temporal gyrus [23].

\section{Acknowledgment}

This work was funded by grants from the Michael J Fox Foundation for Parkinson's Research, the W Garfield Weston Foundation, and the Alzheimer's Association, the Canadian Institutes for Health Research, and the Natural Sciences and Engineering Research Council of Canada. We thank Christian Beckmann and Simon Eickhoff for their advice on data analysis. Data used in this article were obtained from the Parkinsons Progression Markers Initiative (PPMI) database (www.ppmi-info.org/data). For up-to-date information on the study, visit www.ppmi-info.org. PPMI is sponsored and partially funded by the Michael J Fox Foundation for Parkinsons Research and funding partners, including AbbVie, Avid Radiopharmaceuticals, Biogen, Bristol-Myers Squibb, Covance, GE Healthcare, Genentech, GlaxoSmithKline (GSK), Eli Lilly and Company, Lundbeck, Merck, Meso Scale Discovery (MSD), Pfizer, Piramal Imaging, Roche, Servier, and UCB (www.ppmi-info.org/fundingpartners). 


\section{References}

1. Antal, A., Bandini, F., Kéri, S., Bodis-Wollner, I.: Visuo-cognitive dysfunctions in parkinson's disease. Clinical Neuroscience (New York, NY) 5(2) (1997) 147-152

2. Armstrong, R.A.: Visual symptoms in parkinson's disease. Parkinson's disease 2011 (2011)

3. Armstrong, R.A.: Visual signs and symptoms of parkinson's disease. Clinical and Experimental Optometry 91(2) (2008) 129-138

4. Le Bihan, D.: Looking into the functional architecture of the brain with diffusion mri. Nature Reviews Neuroscience 4(6) (2003) 469-480

5. Meppelink, A.M., de Jong, B.M., Renken, R., Leenders, K.L., Cornelissen, F.W., van Laar, T.: Impaired visual processing preceding image recognition in parkinson's disease patients with visual hallucinations. Brain 132(11) (2009) 2980-2993

6. Wheeler-Kingshott, C., Trip, S., Symms, M., Parker, G., Barker, G., Miller, D.: In vivo diffusion tensor imaging of the human optic nerve: pilot study in normal controls. Magnetic resonance in medicine 56(2) (2006) 446-451

7. Van Der Walt, A., Kolbe, S.C., Wang, Y.E., Klistorner, A., Shuey, N., Ahmadi, G., Paine, M., Marriott, M., Mitchell, P., Egan, G.F., et al.: Optic nerve diffusion tensor imaging after acute optic neuritis predicts axonal and visual outcomes. PLoS One 8(12) (2013) e83825

8. Aarabi, M.H., Kamalian, A., Mohajer, B., Shandiz, M.S., Eqlimi, E., Shojaei, A., Safabakhsh, H.: A statistical approach in human brain connectome of parkinson disease in elderly people using network based statistics. In: 2015 37th Annual International Conference of the IEEE Engineering in Medicine and Biology Society (EMBC), IEEE (2015) 4310-4313

9. Yu, C., Lin, F., Li, K., Jiang, T., Zhu, C., Qin, W., Sun, H., Chan, P.: Diffusion tensor imaging in the assessment of normal-appearing brain tissue damage in relapsing neuromyelitis optica. American Journal of Neuroradiology 27(5) (2006) 1009-1015

10. Yu, C.S., Zhu, C.Z., Li, K.C., Xuan, Y., Qin, W., Sun, H., Chan, P.: Relapsing neuromyelitis optica and relapsing-remitting multiple sclerosis: Differentiation at diffusion-tensor mr imaging of corpus callosum 1. Radiology 244(1) (2007) 249-256

11. Kolbe, S., Chapman, C., Nguyen, T., Bajraszewski, C., Johnston, L., Kean, M., Mitchell, P., Paine, M., Butzkueven, H., Kilpatrick, T., et al.: Optic nerve diffusion changes and atrophy jointly predict visual dysfunction after optic neuritis. Neuroimage 45(3) (2009) 679-686

12. Li, M., Li, J., He, H., Wang, Z., Lv, B., Li, W., Hailla, N., Yan, F., Xian, J., Ai, L.: Directional diffusivity changes in the optic nerve and optic radiation in optic neuritis. The British journal of radiology (2014)

13. Li, J., Shi, W., Li, M., Wang, Z., He, H., Xian, J., Lv, B., Yan, F.: Time-dependent diffusion tensor changes of optic nerve in patients with indirect traumatic optic neuropathy. Acta Radiologica 55(7) (2014) 855-863

14. Marek, K., Jennings, D., Lasch, S., Siderowf, A., Tanner, C., Simuni, T., Coffey, C., Kieburtz, K., Flagg, E., Chowdhury, S., et al.: The parkinson progression marker initiative (ppmi). Progress in neurobiology 95(4) (2011) 629-635

15. Leemans, A., Jeurissen, B., Sijbers, J., Jones, D.: Exploredti: a graphical toolbox for processing, analyzing, and visualizing diffusion mr data. In: 17th Annual Meeting of Intl Soc Mag Reson Med. Volume 209. (2009) 3537 
16. Collier, Q., Veraart, J., Jeurissen, B., den Dekker, A.J., Sijbers, J.: Iterative reweighted linear least squares for accurate, fast, and robust estimation of diffusion magnetic resonance parameters. Magnetic resonance in medicine $\mathbf{7 3 ( 6 )}$ (2015) $2174-2184$

17. Rohde, G., Barnett, A., Basser, P., Marenco, S., Pierpaoli, C.: Comprehensive approach for correction of motion and distortion in diffusion-weighted mri. Magnetic resonance in medicine 51(1) (2004) 103-114

18. Leemans, A., Jones, D.K.: The b-matrix must be rotated when correcting for subject motion in dti data. Magnetic Resonance in Medicine 61(6) (2009) 13361349

19. Tzourio-Mazoyer, N., Landeau, B., Papathanassiou, D., Crivello, F., Etard, O., Delcroix, N., Mazoyer, B., Joliot, M.: Automated anatomical labeling of activations in spm using a macroscopic anatomical parcellation of the mni mri single-subject brain. Neuroimage 15(1) (2002) 273-289

20. Zalesky, A., Fornito, A., Bullmore, E.T.: Network-based statistic: identifying differences in brain networks. Neuroimage 53(4) (2010) 1197-1207

21. Goetz, C.G., Vaughan, C.L., Goldman, J.G., Stebbins, G.T.: I finally see what you see: Parkinson's disease visual hallucinations captured with functional neuroimaging. Movement Disorders 29(1) (2014) 115-117

22. Yao, N., Shek-Kwan Chang, R., Cheung, C., Pang, S., Lau, K.K., Suckling, J., Rowe, J.B., Yu, K., Ka-Fung Mak, H., Chua, S.E., et al.: The default mode network is disrupted in parkinson's disease with visual hallucinations. Human brain mapping 35(11) (2014) 5658-5666

23. Zhang, J., Bi, W., Zhang, Y., Zhu, M., Zhang, Y., Feng, H., Wang, J., Zhang, Y., Jiang, T.: Abnormal functional connectivity density in parkinson's disease. Behavioural brain research 280 (2015) 113-118 